\title{
Disturbance Observer Structure Applied to Sensorless Brushless DC Motors Drive
}

\author{
Samuel Wang, Chin-Hsuan Lu, and An-Chen Lee
}

\begin{abstract}
In a permanent magnet (PM) brushless dc motor (BLDC), the performance of Hall effect sensors are not reliable in some applications. The back electromotive force (back-EMF) signal is used instead since the zero-crossing points of back-EMF can be used to provide the rotor position information. In this paper, a new back-EMF difference detection method based on disturbance observer is proposed. The proposed structure can detect the back-EMF as well as back-EMF difference signal. The zero-crossing points of the back-EMF difference can be used directly for commutation, requiring no phase shift. Simulation and experiment results are provided to show the feasibility of the proposed method.
\end{abstract}

Index Terms-Back electromotive force (EMF), brushless DC motor, disturbance observer, sensorless.

\section{INTRODUCTION}

Brushless DC (BLDC) motors were extensively used in recent years due to its high efficiency and high reliability. In general, BLDC motors use Hall sensors to detect the corresponding position. However, in some extremely industrial applications, such as high temperature surrounding, small size, and economical limitation, it is not suitable to apply Hall sensors. For these reasons, many researches pay attention to the drive schemes of sensorless BLDC motor.

Many papers have presented several sensorless drive schemes for BLDC motors. These papers can be classified into three categories [1], i.e., direct back-EMF detection, indirect back-EMF detection and techniques based on estimation and models. Direct back-EMF detection method senses the back-EMF of the floating phase and its zero crossing points are used to trigger the commutation instants. P. Damodharan and K. Vasudevan proposed a sensorless drive that detects back-EMF from line voltage difference [2]. T. Kim et al. proposed another method based on terminal voltage difference. Unlike the conventional one, the method uses three additional comparing circuits to detect the back-EMF difference to eliminate phase delay [3]. The other papers concern the issue that the motor speed range is narrow due to the phase delay problem caused by filtering. Therefore, indirect back-EMF detection method are

Manuscript received March 10, 2014; revised May 8, 2014. This work was supported by the National Science Council of the Republic of China, under Contract NSC 102-2221-E-009-052-MY2.

Samuel Wang and An-Chen Lee are with the National Chiao Tung University, Hsinchu, Taiwan (e-mail: samuel6113@ hotmail.com.tw, aclee@mail.nctu.edu.tw).

Chin-Hsuan Lu was with National Chiao Tung University, Hsinchu, Taiwan. $\mathrm{He}$ is with the DELTA, Taoyuan, Taiwan (e-mail: bn31007@gmail.com). proposed. J. C. Moreira proposed a method that can extract third harmonic component from the stator phase voltage while the fundamental and other polyphase component are eliminated via a simple summation of three phase voltage [4]. S. Ogasawara proposed a sensorless drive based on conducting state of free-wheeling diodes connected in antiparallel with power transistors [5]. Some researchers also proposed techniques based on model-based estimation. Z. M. A. Peixoto et al. apply sliding mode observer to estimate back-EMF, rotor position and speed [6]. R. Dhaouadi et al. used extended Kalman filter to estimate speed and rotor position with the information of motor voltages and currents [7].

The disturbance observer (DOB) structure which reduces sensitivity to modeling error and enhances disturbance rejection properties is widely used in the field of motion control. The DOB is often applied to the inner-loop of the motion control structure to reject equivalent disturbance as much as possible for achieving precise position tracking. It was first introduced by Ohnishi [8] and refined by Umeno and Hori [9].

This paper presents a new model-based sensorless drive based on back-EMF difference detection method. Consider the back-EMF difference as input disturbance, the DOB structure can be applied on BLDC motor. In addition, the proposed structure can detect the back-EMF as well as back-EMF difference signal. The main advantage of this method is that the back-EMF difference does not require any phase shift from zero-crossing points; the algorithm is thus simpler and the performance is better.

The organization of this paper is as follows. Section II describes the mathematical model of BLDC motors. Section III discussed the proposed sensorless drive. Section IV demonstrates the simulation results. Section V shows the results of the experiment. Section VI presents the conclusion.

\section{MODEL OF BLDC MOTOR}

The BLDC motor model and the three phase inverter are shown in Fig. 1. The three IGBTs, $T_{1}, T_{2}$ and $T_{3}$, in the upper side of the inverter are called high side transistors in this paper; the other three IGBTs are called low side transistors. Assuming that the stator resistances in the three windings are identical and the mutual inductances are constant, the phase voltage equation is shown in (1)

$$
\left[\begin{array}{l}
V_{a n} \\
V_{b n} \\
V_{c n}
\end{array}\right]=\left[\begin{array}{ccc}
R & 0 & 0 \\
0 & R & 0 \\
0 & 0 & R
\end{array}\right]\left[\begin{array}{l}
i_{a} \\
i_{b} \\
i_{c}
\end{array}\right]+\left[\begin{array}{ccc}
L & 0 & 0 \\
0 & L & 0 \\
0 & 0 & L
\end{array}\right] \frac{d}{d t}\left[\begin{array}{l}
i_{a} \\
i_{b} \\
i_{c}
\end{array}\right]+\left[\begin{array}{l}
e_{a} \\
e_{b} \\
e_{c}
\end{array}\right]
$$

where $V_{a n}, V_{b n}$ and $V_{c n}$ are phase voltages, $e_{a}, e_{b}$ and $e_{c}$ are 
back-EMFs of each phase, $R$ is the phase resistance, and $L$ is the equivalent inductance in the stator windings.

Fig. 2 illustrates the ideal relationship between back-EMF and current waveform. Only two phases will be conducted at the same interval, $(0+60 k)$ electrical degrees, where $k=0,1$, $2 \ldots$, and the third phase will be floating. The back-EMF waveform provides the information of the rotor position with zero-crossing points at $(0+30 k)$ electrical degrees. In conventional sensorless drive, 30 -degree phase shift from the zero-crossing points of the back-EMF signal can be utilized for commutation.

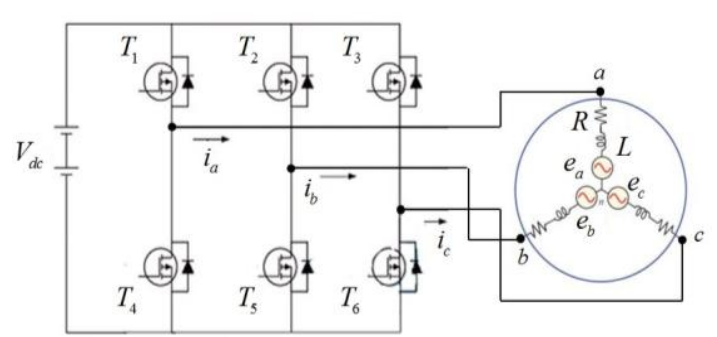

Fig. 1. BLDC motor and three phase inverter.

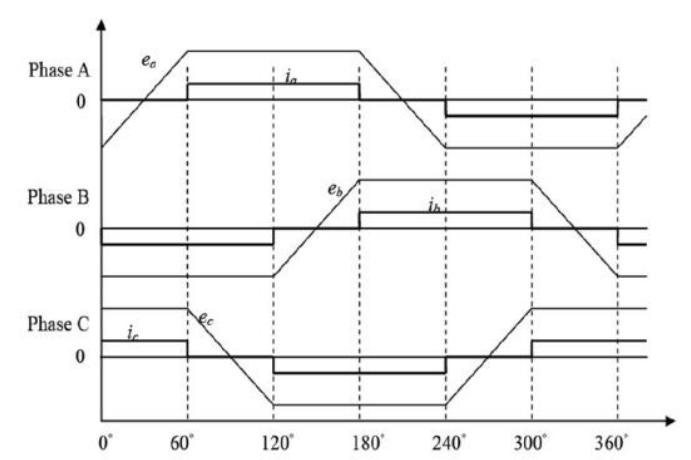

Fig. 2. Relationship between back-EMF and current waveform.

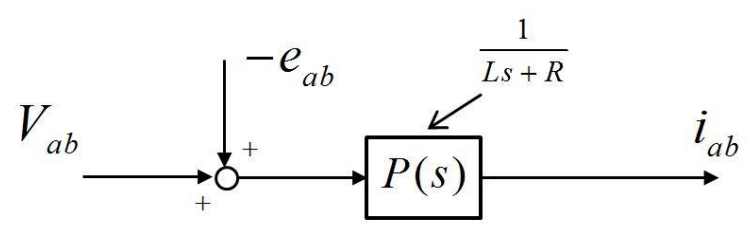

Fig. 3. Block diagram of equation 4.

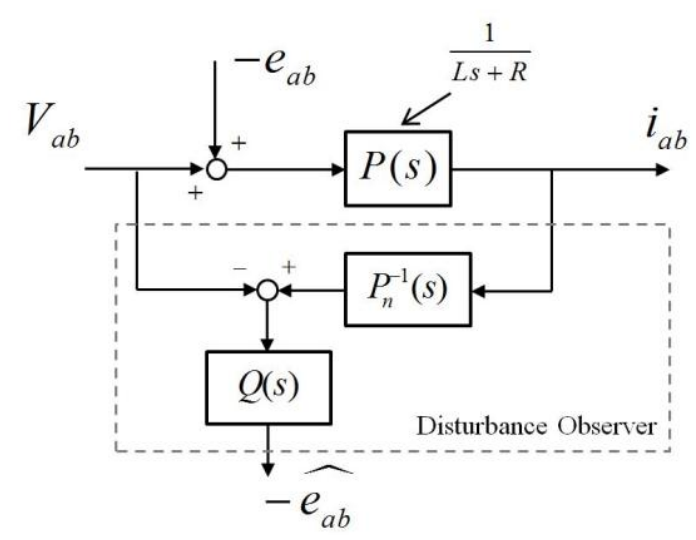

Fig. 4. back-EMF detection method based on disturbance observer.

\section{PROPOSED SENSORLESS DRIVE}

This paper proposed a new back-EMF detection method based on DOB. The estimated disturbance in the DOB structure is not used for compensation but used to detect the
back-EMF difference. The following mathematical derivation illustrates how the DOB structure is applied on the BLDC motor.

Three line voltage equations can be defined as

$$
\begin{aligned}
& V_{a b}=V_{a n}-V_{b n} \\
& V_{b c}=V_{b n}-V_{c n} . \\
& V_{c a}=V_{c n}-V_{a n}
\end{aligned}
$$

From (1) and (2), the line voltage equations are given as

$$
\begin{aligned}
& V_{a b}=R i_{a b}+L \frac{d\left(i_{a b}\right)}{d t}+e_{a b} \\
& V_{b c}=R i_{b c}+L \frac{d\left(i_{b c}\right)}{d t}+e_{b c} \\
& V_{c a}=R i_{c a}+L \frac{d\left(i_{c a}\right)}{d t}+e_{c a}
\end{aligned}
$$

Line voltages are used to eliminate the influence of the neutral voltage part because the neutral voltage changes with the PWM switching signal, which makes conventional phase voltage and terminal voltage drive more complicated. Taking Laplace transform of (3) gives

$$
\begin{aligned}
& V_{a b}(s)=R i_{a b}(s)+L s i_{a b}(s)+e_{a b}(s) \\
& V_{b c}(s)=R i_{b c}(s)+L s i_{b c}(s)+e_{b c}(s) \\
& V_{c a}(s)=R i_{c a}(s)+L s i_{c a}(s)+e_{c a}(s)
\end{aligned}
$$

Consider the line voltages as input, line currents as output and the back-EMF difference as the input disturbance, the block diagram of (4) is obtained in Fig. 3, where the voltage and current can be measured from sensors and the parameters of the plant, resistance and inductance, are provided from the datasheet. With this setup, the DOB structure can be applied on BLDC motor, as shown in Fig. 4, for estimating the back-EMF difference. The estimated back-EMF difference signal can be derived as

$$
-\hat{e}_{a b}=-V_{a b} Q(\mathrm{~s})+\left(V_{a b}-e_{a b}\right) P(s) P_{n}^{-1}(s) Q(s) .
$$

If no measurement noise or modeling error is involved, such that $P(\mathrm{~s})=P_{n}(\mathrm{~s}), e_{a b}$ can be obtained as

$$
-\hat{e_{a b}}=-e_{a b} Q(s)
$$

Since the back-EMF difference is mostly composed of low frequency component, the $Q$ here is a first-order low pass filter used to yield an implementable proper rational function and minimize the influence from sensor noise, as shown below.

$$
Q(s)=\frac{\tau}{s+\tau}
$$

where $\tau$ is the cutoff frequency of the filter. The proposed structure is thus being modified into Fig. 5. The other two 
back-EMF difference, $e_{b c}$ and $e_{c a}$ can also be obtained from different line voltage input and line current output, where

$$
\begin{aligned}
& e_{a b}(\mathrm{~s})=e_{a}(\mathrm{~s})-e_{b}(\mathrm{~s}) \\
& e_{b c}(\mathrm{~s})=e_{b}(\mathrm{~s})-e_{c}(\mathrm{~s}) \\
& e_{c a}(\mathrm{~s})=e_{c}(\mathrm{~s})-e_{a}(\mathrm{~s})
\end{aligned}
$$

As one can see from (8), the three equations are linear dependent, and thus one more property is needed to solve three back-EMFs. During any conducting interval, since only two phases are conducting and the third one is floating, the back-EMFs of the two conducting phases, the flat area in Fig. 2 , are equal and opposite. Hence, the back-EMFs in (8) can be solved. However, the back-EMFs are not used in this work because the zero-crossing points of back-EMF difference can be used directly for commutation. The ideal waveform of the estimated back-EMF difference is shown in Fig. 6. Table I provides the commutation trigger criteria.

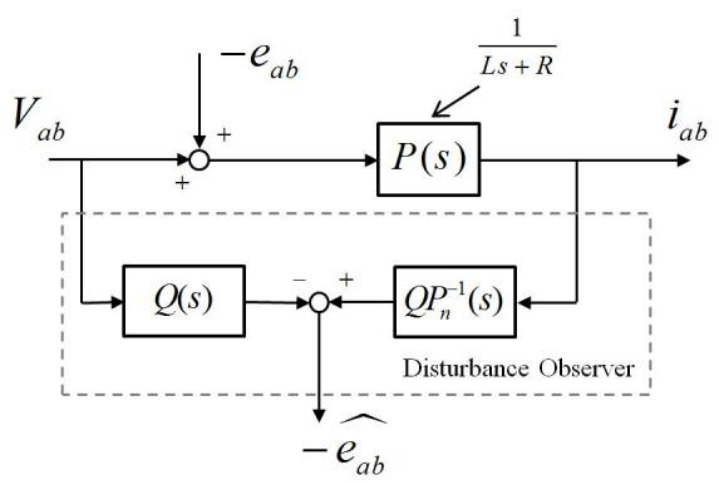

Fig. 5. Modified disturbance observer structure for implementation.

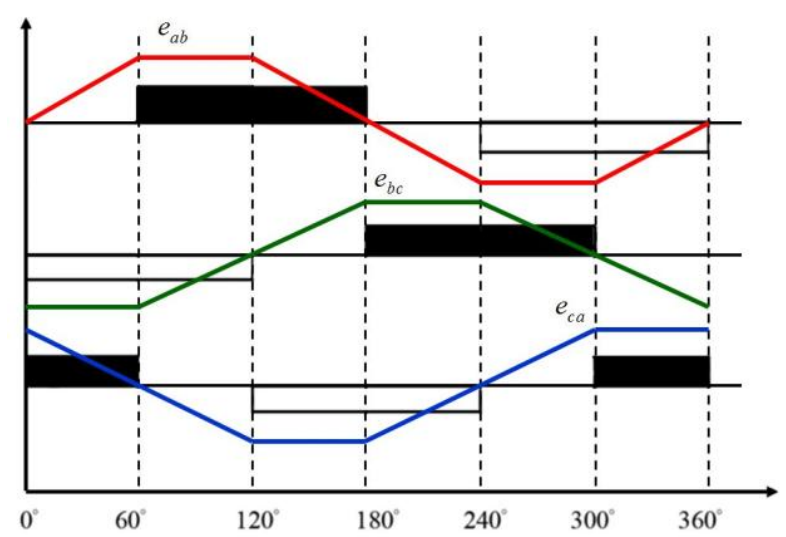

Fig. 6. Ideal back-EMF difference waveform and H_PWM_L_ON mode.

TABLE I: COMMUTATION TRIGGER CRITERIA

\begin{tabular}{|c|c|c|c|}
\hline Angle & $0^{\circ}-60^{\circ}$ & $60^{\circ}-120^{\circ}$ & $120^{\circ}-180^{\circ}$ \\
\hline $\begin{array}{c}\text { Trigger } \\
\text { Criteria }\end{array}$ & $e_{c a}=0$ & $e_{b c}=0$ & $e_{a b}=0$ \\
\hline $\begin{array}{c}\text { Conducting } \\
\text { Transistors }\end{array}$ & $T_{3} T_{5}$ & $T_{1} T_{5}$ & $T_{1} T_{6}$ \\
\hline
\end{tabular}

\begin{tabular}{|c|c|c|c|}
\hline Angle & $180^{0}-240^{\circ}$ & $240^{\circ}-300^{\circ}$ & $300^{0}-360^{0}$ \\
\hline $\begin{array}{c}\text { Trigger } \\
\text { Criteria }\end{array}$ & $e_{c a}=0$ & $e_{b c}=0$ & $e_{a b}=0$ \\
\hline $\begin{array}{c}\text { Conducting } \\
\text { Transistors }\end{array}$ & $T_{2} T_{6}$ & $T_{2} T_{4}$ & $T_{3} T_{4}$ \\
\hline
\end{tabular}

With two commutation points in each phase, the proposed back-EMF detection method provides the rotor position information with 60 degree resolution. Note that the 30-degree phase shift from zero-crossing points in conventional sensorless drive is not required. The disadvantage of conventional ones is that it should calculate time span for 30-degree phase shift from one complete electrical cycle. The need for 30-degree phase shift from zero-crossing points will increase the complexity of the algorithm and the inaccuracy of the commutation points under speed fluctuation.

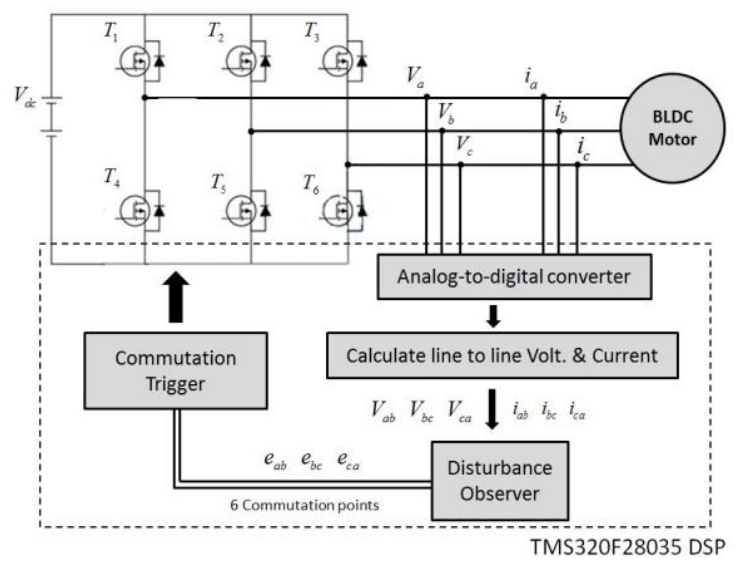

Fig. 7. Overall structure of the proposed sensorless drive.

The proposed sensorless drive will be demonstrated under H_PWM_L_ON mode, as shown in Fig. 6, i.e. using PWM mode on the high side transistors and keeping constant turn-ON mode on the low side transistors. Forced alignment is used as starting procedure; once the rotor starts rotating, back-EMF differences are being detected by the DOB structure. Overall structure of the proposed sensorless drive is shown in Fig. 7. Phase voltage and current are measured from sensors and read through the analog-to digital converter. The DOB structure provides six commutation points to the three phase inverter.

\section{Simulation of the Proposed Method}

Simulations are presented to show the feasibility of the proposed method. Matlab Simulink is used in this work. For simplicity, the initial rotor position is provided in starting procedure since the starting technique is not discussed in this paper. After the rotor starts rotating, the commutation information is switched to the source of the proposed method. Fig. 8 shows the layout of the BLDC motor structure; it consists of a dc source, a three phase inverter with six IGBTs and a permanent magnet synchronous machine.

In this paper, the experiment prototype uses voltage divider and filter to measure phase voltages and shunt resistors to sense currents. The Q filter is designed in accord with the hardware implementation; the cut off frequency is set to 2360 and the dc gain is set to 0.0081, as shown in Fig. 9. The relationship between the real back-EMF difference and the estimated back-EMF difference under $600 \mathrm{rpm}$ and 1500 rpm is shown in Figs. 10 and 11. As one can discover from the results, the estimated result lags the real signal. This delay comes from the filter. As the speed increase, the magnitude of 
phase delay becomes larger. Since the magnitude can be calculated from the frequency response of the filter, the phase delay during commutation is being compensated.

\section{EXPERIMENT RESULTS}

Experiments are performed to verify the proposed sensorless drive. The experiment prototype, shown in Fig. 12, consists of a BLDC motor and PFC Kit from Texas Instrument with Digital Signal Processor (DSP) TMS320F28035.

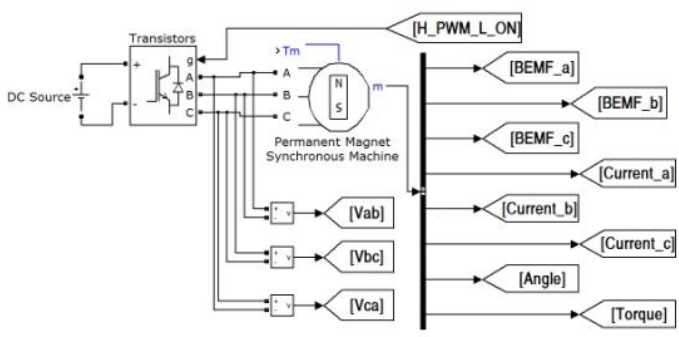

Fig. 8. BLDC motor structure in simulation.

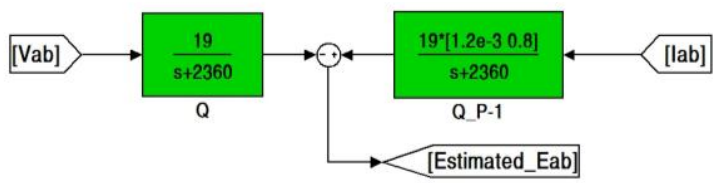

Fig. 9. Disturbance observer structure in simulation.

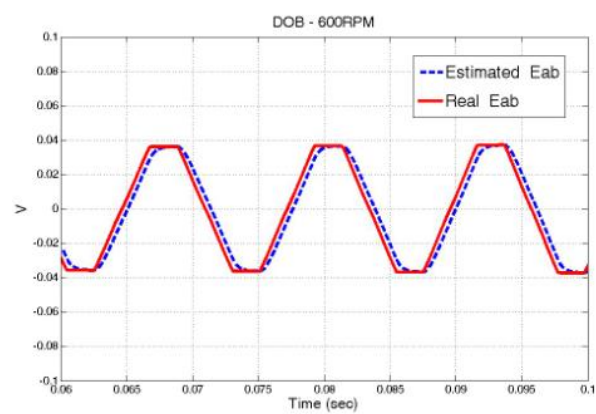

Fig. 10. Simulation results at $600 \mathrm{rpm}$.

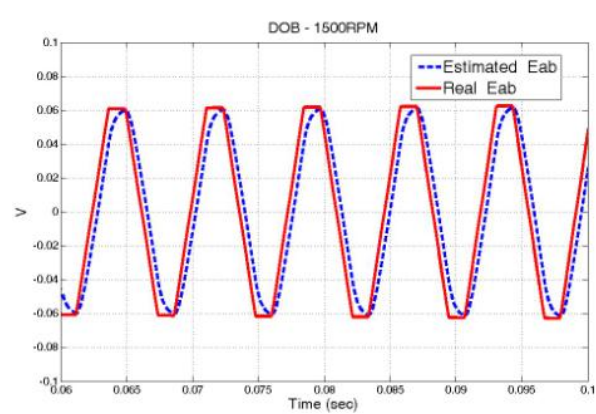

Fig. 11. Simulation results at $1500 \mathrm{rpm}$.

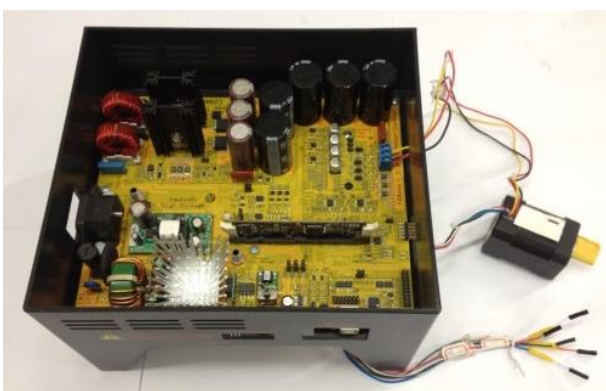

Fig. 12. Experimental prototype.

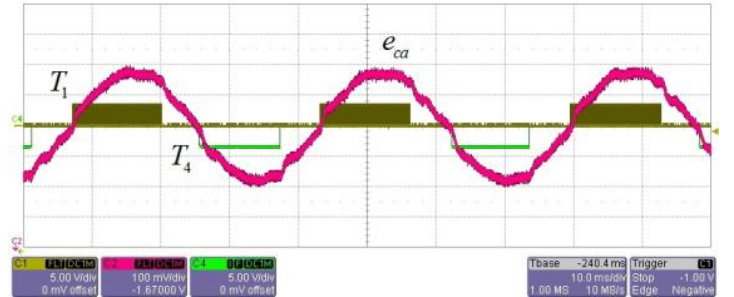

Fig. 13. back-EMF difference and driving signal.

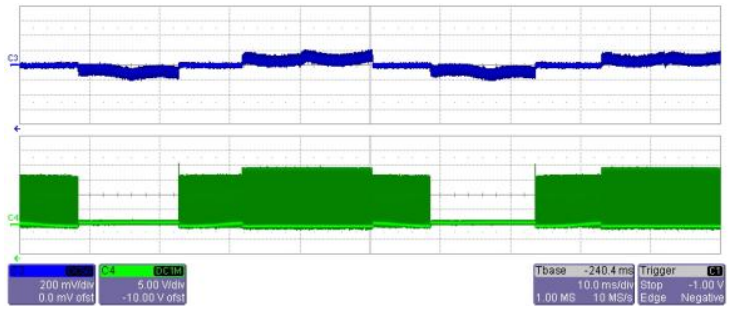

(a). $300 \mathrm{rpm}$.

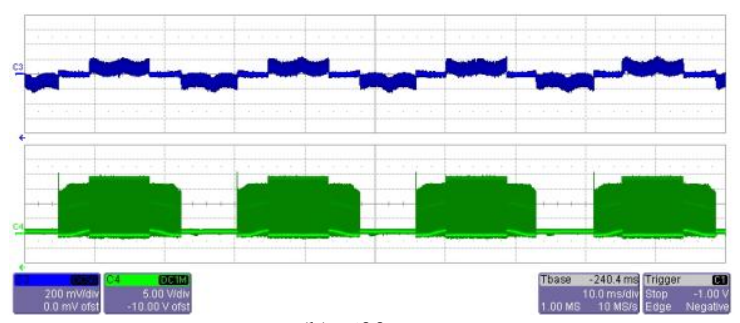

(b). $600 \mathrm{rpm}$

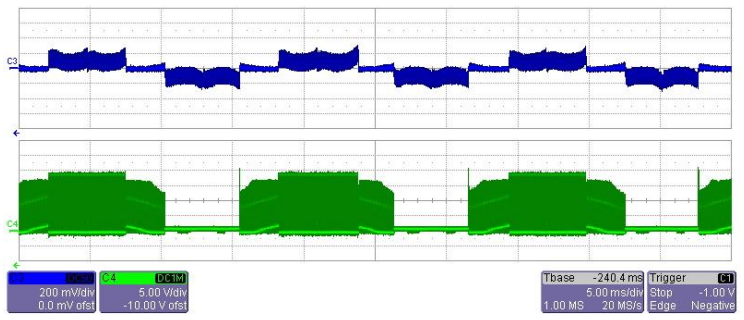

(c). $1000 \mathrm{rpm}$

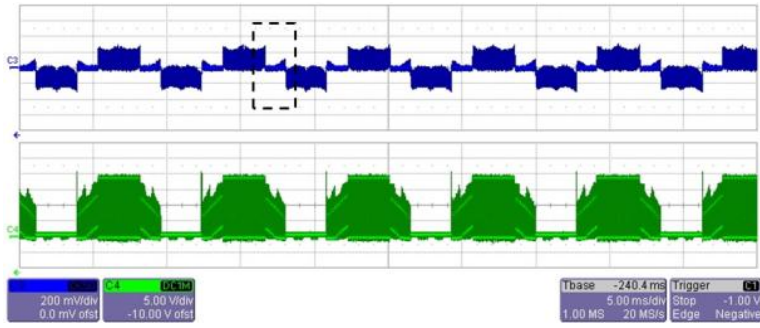

(d). $2000 \mathrm{rpm}$

Fig. 14. Experiment result of the proposed sensorless drive.

TABLE II: PARAMETERS OF THE BLDC MOTOR
\begin{tabular}{|l|l|}
\hline Rated Voltage & $24(\mathrm{~V})$ \\
\hline Rated Power & $53($ Watts $)$ \\
\hline Rated Speed & $4000(\mathrm{rpm})$ \\
\hline Resistance & $0.8(\mathrm{ohm})$ \\
\hline Inductance & $0.0012(\mathrm{H})$ \\
\hline back-EMF Voltage & $4.14(\mathrm{~V} / \mathrm{k} \mathrm{rpm})$ \\
\hline Number of Poles & 8 \\
\hline
\end{tabular}

The motor is driven by a three phase inverter consist of six IGBTs. The motor parameters are shown in Table II.

Fig. 13 shows the back-EMF difference signal detected by the DOB structure and the driving signal of the three phase inverter of phase A. As shown in Fig. 13, the zero-crossing points are used directly for commutation. The black part of the driving signal is the PWM switching signal for high side 
transistors, while the white part is the constant ON signal for low side transistors.

Current waveform and terminal voltage of phase A are shown in Fig. 14. The current waveform from the experiment matches the ideal rectangular waveform shown in Fig. 2. The current ripple during the non-conducted period, indicated in Fig. 14 (d), takes place when the current flows through the antiparallel diode during PWM off time. This effect results in speed fluctuation and produces acoustic noise and vibration, which is undesirable in machine tool or motion control applications. The elimination of current ripple requires further PWM or control techniques, which is beyond the scope of this paper.

\section{CONCLUSIONS}

In this paper, a new back-EMF difference detection method is presented. The proposed method applies the DOB structure on BLDC motors. The estimated back-EMF difference is used for commutation directly without any phase shift. The simulation and experiment results successfully match the theories in this paper.

\section{REFERENCES}

[1] J. C. Gamazo-Real, E. Vázquez-Sánchez, and J. Gómez-Gil, "Position and speed control of brushless DC motors using sensorless techniques and application trends," Sensors, vol. 10, pp. 6901-6947, 2010.

[2] P. Damodharan and K. Vasudevan, "Sensorless brushless DC motor drive based on the zero-crossing detection of back electromotive force (EMF) from the line voltage difference," IEEE Trans. Energy Conver., vol. 25, pp. 661-668, 2010.

[3] K. Taeyeon, K. Chungil, and L. Joon, "A new sensorless drive scheme for a BLDC motor based on the terminal voltage difference," in Proc. IEEE. Ind. Electron. Society Conf. , 2011, pp. 1710-1715.

[4] J. C. Moreira, "Indirect sensing for rotor flux position of permanent magnet AC motors operating over a wide speed range," IEEE Trans. Ind. Appl., vol. 32, pp. 1394-1401, 1996.

[5] S. Ogasawara and H. Akagi, "An approach to position sensorless drive for brushless DC motors," IEEE Trans. Ind. Appl., vol. 27, pp. 928-933, 1991.

[6] Z. M. A. Peixo, F. M. F. Sa, P. F. Seixas, B. R. Menezes, P. C. Cortizo, and W. S. Lacerda, "Application of sliding mode observer for induced e.m.f., position and speed estimation of permanent magnet motors," in Proc. Int. Conf. Power Electron. Drive Syst., pp. 599-604, vol. 2, 1995.
[7] R. Dhaouadi, N. Mohan, and L. Norum, "Design and implementation of an extended Kalman filter for the state estimation of a permanent magnet synchronous motor," IEEE Trans. Power Electron., vol. 6, pp. 491-497, 1991.

[8] K. Ohishi, M. Nakao, K. Ohnishi, and K. Miyachi, "Microprocessor-controlled DC motor for load-in sensitive position servo system," IEEE Trans. Ind. Electron., vol. 34, pp. 44-49, Feb. 1987.

[9] T. Umeno and Y. Hori, "Robust speed control of DC servomotors using modern two degrees-of-freedom controller design," IEEE Trans. Ind. Electron., vol. 38, pp. 363-368, 1991.

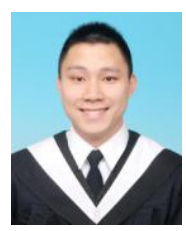

Samuel Wang was born in California, USA, in 1990. He received the B.S. degree in mechanical engineering from National Chiao Tung University, Hsinchu, Taiwan in 2012 and is currently pursuing the M.S. degree in mechanical engineering from National Chiao Tung University, Hsinchu, Taiwan.

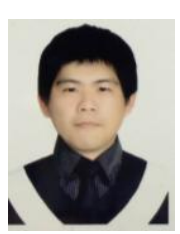

Chin-Hsuan Lu was born in Taipei, Taiwan, in 1988. He received the B.S. degree from National Taiwan Ocean University, Keelung, Taiwan, and M.S. degree from National Chiao Tung University, Hsinchu, Taiwan, both in mechanical engineering, in 2011 and 2013, respectively. $\mathrm{He}$ is currently working at the DELTA, Taoyuan, Taiwan.

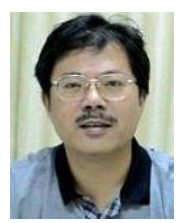

An-Chen Lee was born in Chiayi, Taiwan in 1956. He received the B.S. and M.S. degrees in power mechanical engineering from National Tsing Hua University, Hsinchu, Taiwan and the Ph.D. degree in 1986 from University of Wisconsin-Madison in Mechanical Engineering, USA.

$\mathrm{He}$ is designated as a chair professor of National Chiao Tung University and currently a professor in the Department of Mechanical Engineering. His current research interests are CNC machine tool control technology, magnetic bearing technology, rotor dynamic and control, and semiconductor manufacturing process control. Prof. Lee served as an editorial board member of International Journal of Precision Engineering and Manufacturing, Chinese society of Mechanical Engineers. He is the recipient of National Science Committee (NSC) Excellent Research Award (1991-1992), NSC Distinguished Research Award (1993-1994, 1995-1996, 1997-1998), NSC research fellow (1999-2001, 2002-2004), NSC research fellow Award (2005), Chinese Society of Mechanical Engineers Distinguished Engineering Professor Award (2001), Gold Medal Award, Inventor/New Product Exposition (INPEX), Pittsburgh, U.S.A. (2010), Gold Medal Award, International Exhibition of Inventions of Geneva (2011), Brown Medal Award, International Exhibition of Inventions of Geneva (2012). 